\title{
Wavelet-based acoustic emission characterization of damage mechanism in composite materials under mode I delamination at different interfaces
}

\author{
A. R. Oskouei, M. Ahmadi*, M. Hajikhani
}

Non-destructive Testing Lab., Department of Mechanical Engineering, Amirkabir University of Technology, 424 Hafez Ave., 15914, Tehran, Iran

\begin{abstract}
In this paper, acoustic emission (AE) monitoring with a wavelet-based signal processing technique is developed to detect the damage types during mode I delamination on glass/polyester composite materials. Two types of specimen at different midplane layups, woven/woven (T3) and unidirectional/unidirectional (T5), leading to different levels of damage evolution, were studied. Double cantilever beam (DCB) is applied to simulate delamination process for all specimens. Firstly, the obtained AE signals are decomposed into various wavelet levels. Each level includes detail and approximation that are called components and related to a specific frequency range. Secondly, the energy distribution criterion is applied to find the more significant components each one of which is in relation to a distinct type of damage. The results show that the energy of AE signals has been concentrated in three significant components for both of the specimens. There is a difference in energy distribution of similar components of two specimens. It indicates that there is a dissimilar dominant damage mechanism for two different interfaces during the delamination process. Additionally, the microscopic observation (SEM) is used to determine how the different fracture mechanisms are related to the dominant corresponding wavelet components.
\end{abstract}

Keywords: damage mechanism, polymer composites, delamination, acoustic emission, wavelet packet

\section{Introduction}

The main fracture mode that has an effect on the residual strength of composite materials is delamination. Mode I inter-laminar fracture has received the greatest attention, and various standards have been developed for the double cantilever beam (DCB) test [1].

In order to identify the type of damage in composites, which involves fiber failure, the transverse failure and delamination, the acoustic emission (AE) technique is also promising for the detection of the damage type [2]. This technique is based on the detection of elastic surface stress waves caused by the dissipation of elastic energy due to the opening of a crack or plastic deformation. Several stud- ies were already conducted on the mode I fracture to determine the surface energies of composite materials by creating a crack propagation effect $[3,4]$.

To find correlation between AE parameters and damage mechanism several studies have been carried out [5-9]. Most studies so far have used AE descriptors such as counts, amplitude, energy and also multiparameter method to characterize the development of failure mechanisms. In this way, Siron et al. [10] used AE waveform parameters to investigate damage indicators which are related to the physical damage of the composite. RamirezJimenez et al. [11] have applied the frequency method to identify the failure modes in glass/ 
polypropylene composites. The results showed that $100 \mathrm{kHz}$ primary event frequency is due to the fiber/matrix debonding, those between 200 and $300 \mathrm{kHz}$ are due to the fiber slippage and fiber pull out and the two higher frequencies appearing in all tests are related to the fiber breaking. Similar results are obtained by Haselbach and Lauke [12] for evaluating the debonding between fiber and matrix. It was found that debonding is accompanied by signals showing the amplitudes smaller than $45 \mathrm{~dB}$ and conspicuous frequencies above $250 \mathrm{kHz}$ at one or more hits of an event.

Many of the useful information get averaged/lost, while transforming a signal from one domain to another. These problems have been successfully eliminated by the introduction of time-frequency distributions. Among them, wavelet transform is one of the most promising methods followed in engineering. $\mathrm{Ni}$ and Iwamoto [13] concluded that using the time-frequency method of the wavelet transform, the microfailure modes at a fiber breakage and the microfracture mechanism, such as the sequence of each failure mode and their interaction, were clearer. Qi and coworkers $[14,15]$ applied the wavelet transform to analyze AE signals by decomposing the signal into different wavelet levels. Each level represents the components of the decomposed AE signal within a certain frequency range. The energy criteria at each level were used to identify the dominant fracture mechanism in an order. A similar work was presented on damage evolution in center-hole glass/polyester composites by Loutas et al. [16].

From the above literature review, it appears that there are several different approaches to the event recognition analysis. In this paper, wavelet packet transform method was applied to provide the relevant information from $\mathrm{AE}$ signal to discriminate the damage types. The AE signals used for this analysis have been measured during quasi-static loading. The energy criterion has been used for analyzing each level of $\mathrm{AE}$ signals which were decomposed by the wavelet method. The energy criterion is used to find the dominant levels that were related to different failure modes. Additionally, wavelet-based method was combined with microscopic observations by SEM to verify the results of the wavelet analysis. This provides the optimal understanding of the occurrence and the evolution of the basic damage mechanisms.

\section{Experimental procedure}

\subsection{Materials and specimen manufacturing}

Standard double cantilever beam (DCB) specimens were prepared according to the ASTM Standard D5528 [1]. The composite used in this study is the polyester resin (unsaturated resin, S. S. P. Co., Iran) reinforced by the E-glass woven and unidirectional fiber (S. S. P. Co., Iran) with the densities and weights of $1.12 \mathrm{~g} / \mathrm{cm}^{3}, 292 \mathrm{~g} / \mathrm{m}^{2}$ and $500 \mathrm{~g} / \mathrm{m}^{2}$, respectively. The laminates were prepared by hand lay-up with compression molding. The starter crack was formed by inserting a Teflon film at mid-thickness during the molding. Two different specimens which have the same lay-up of [Woven/Unidirectional $]_{5 \mathrm{~s}}$ and different in the midplane interfaces are used. In particular the following specimen midplane interface types have been considered: woven/ woven (T3), and unidirectional/unidirectional (T5). The fiber volume fraction was $60 \%$ for all specimens. The loading blocks were bonded at one end of starter crack in two sides to apply the opening force for delamination. The specimens were prepared with a length of $250 \mathrm{~mm}$, width of $20 \mathrm{~mm}$, thickness of $5 \mathrm{~mm}$, and a starter crack has a length of $70 \mathrm{~mm}$ and the width of $20 \mathrm{~mm}$.

\subsection{Test}

The tests were carried out in a universal test machine (Hiwa Co., Tehran, Iran) with the load cell capacity of $1000 \mathrm{~N}$ at the cross head speed of $0.2 \mathrm{~mm} / \mathrm{min}$. Five samples for each laminate lay-up were used for DCB testing. Each specimen was equipped with an acoustic emission sensor at one end of DCB specimen (Figure 1). The acoustic emission software AEWin and a data acquisition system (PAC) PCI-2 with a maximum sampling rate of $40 \mathrm{MHz}$ were used for recording $\mathrm{AE}$ events. A broadband, resonant-type, single-crystal piezoelectric transducer from Physical Acoustics Corpo-

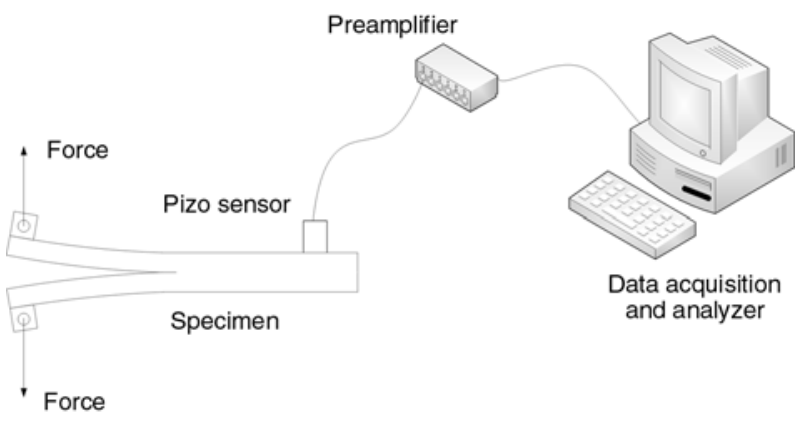

Figure 1. DCB test setup 
ration (PAC), called PICO, was used as the AE sensor. The sensor had a resonance frequency of $513.28 \mathrm{kHz}$, and an optimum operating range of $100-750 \mathrm{kHz}$. AE activities were detected by sensor and amplified by the preamplifier with $40 \mathrm{~dB}$ gain. To improve the signals transmission between specimens and sensor, vacuum silicon grease is used. For avoiding background noise during the sampling, the threshold level was set in $35 \mathrm{~dB}$.

\section{Wavelet transform}

Wavelet transform (WT) has been used as a new method in signal and image processing in last two decades. Some researcher applied the wavelet transform method to analyze acoustic emission (AE) signals [13-17]. Wavelet is a waveform with an effectively limited duration and zero average value. Mathematically, consider a function $\psi$ with the following properties [18-20]:

1. The function integrates with zero (Equation (1)):

$$
\int_{-\infty}^{-\infty} \psi(t) \mathrm{d} t=0
$$

2. It is square integrable or, equivalently, has finite energy (Equation (2)):

$$
\int_{-\infty}^{+\infty}|\psi(t)|^{2} \mathrm{~d} t<\infty
$$

The function $\psi(t)$ is called a mother wavelet or wavelet if it satisfies these two properties as obtained from Equations (1), (2).

Let $f(t)$ be any square integrable function. The CWT or continuous-time wavelet transform of $f(t)$ with respect to a wavelet $\psi$ is defined by Equation (3):

$$
C W T(a, b)=\frac{1}{\sqrt{a}} \int_{-\infty}^{+\infty} f(t) \psi^{*}\left(\frac{t-b}{a}\right) \mathrm{d} t
$$

where the variable $b$ represents time shift or translation, $a$ refers to the scale or dilation variable and * denotes the complex conjugation of wavelet. The inverse continuous wavelet transform can be expressed as [18] (Equations (4), (5)):

$$
f(t)=\frac{1}{C} \int_{a=-\infty}^{+\infty} \int_{b=-\infty}^{+\infty} \frac{1}{|a|^{2}} C W T(a, b) \psi(t) \mathrm{d} a \mathrm{~d} b
$$

$C=\int_{-\infty}^{-\infty} \frac{\left|\psi(\omega)^{2}\right|}{\omega} \mathrm{d} \omega$

Observe that in contrast to Equation (4), which involves $a$ continuum of the dilations and $b$ translations, Equation (6) uses discrete values for these parameters. It means that the dilation takes values of the form $a=2^{j}$ where $j$ is an integer. At any dilation $2^{j}$, the translation parameter takes the values of the form $2^{j} \cdot k$ where $k$ is again an integer [18].

Mathematically, this procedure is described by Equation (6):

$$
f(t)=c \sum_{j} \sum_{k} D W T(j, k) 2^{-j / 2} \psi\left(2^{-j} t-k\right)
$$

The inverse discrete wavelet transform can be expressed by Equation (7):

$$
D W T(j, k)=\int_{-\infty}^{+\infty} f(t) 2^{j / 2} \psi^{*}\left(2^{j} t-k\right) \mathrm{d} t
$$

where $D W T(j, k)$ are the wavelet transform coefficients given by a two-dimensional matrix, $j$ is called the level that is related to the frequency domain of the signal and $k$ is in relation to the time domain. $f(t)$ is the signal that is analyzed and $\psi$ the wavelet used for the analysis.

In the wavelet analysis, a signal is broken apart to an approximation and a detail. The approximations are the low-frequency components of the signal and the details are the high-frequency ones. The approximation is then itself broken apart to a second-level approximation and detail, and the process is repeated to obtain the optimal results [19]. As a result, the signal can be decomposed into a tree structure with wavelet details and wavelet approximations at various levels as shown by Equation (8) [21]:

$$
f(t)=\sum_{i=1}^{i=j} D_{i}(t)+A_{j}(t)
$$

where $D_{i}(t)$ denotes the wavelet detail and $A_{j}(t)$ stands for the wavelet approximation at the $j$ th level, respectively. A graphical representation of DWT of a signal is shown in Figure 2.

The decomposition process of only the approximation component at each level may cause problems while applying DWT in certain applications, where the important information is located in higher frequency components. So, the wavelet packet trans- 


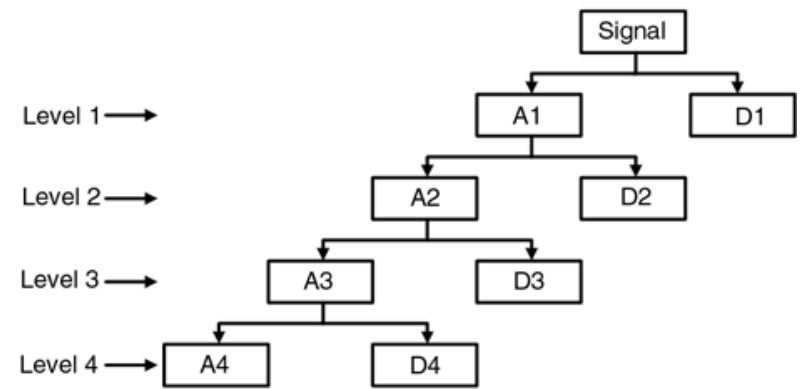

Figure 2. Discrete wavelet transform decomposition tree

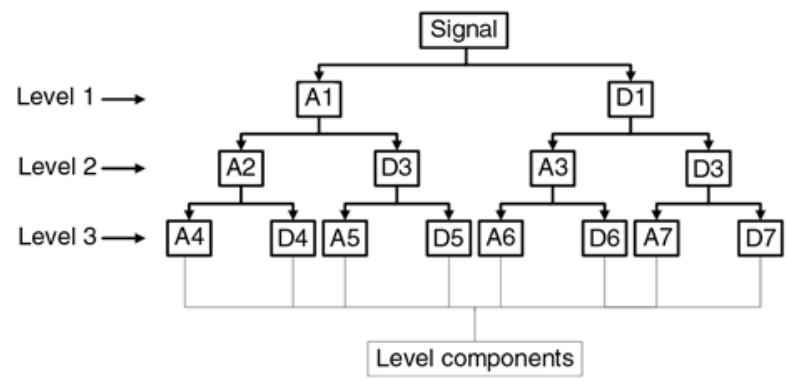

Figure 3. Wavelet packet transform tree

form can resolve this difficulty if a wavelet detail component is also further decomposed in order to obtain its own approximation and detail components (Figure 3). We should note here that in this paper all parts of each level is called component (including details and approximations) and the number of components of level $j$ is equal to $2^{j}$.

A wavelet packet is represented as a function, $\psi_{j, k}^{i}$ where $i$ is the modulation parameter, $j$ is the dilation parameter and $k$ is the translation parameter (Equation (9)):

$\psi_{j, k}^{i}(t)=2^{-j / 2} \psi^{i}\left(2^{-j} t-k\right)$

Here, $i=1,2, \ldots, j^{n}$ and $n$ is the level of decomposition in wavelet packet tree. The wavelet $\psi^{i}$ is obtained through the following recursive relationships (Equations (10) and (11)):

$$
\begin{aligned}
& \psi^{2 i}(t)=\frac{1}{\sqrt{2}} \sum_{k=-\infty}^{+\infty} h(k) \psi^{i}\left(\frac{t}{2}-k\right) \\
& \psi^{2 i+1}(t)=\frac{1}{\sqrt{2}} \sum_{k=-\infty}^{+\infty} g(k) \psi^{i}\left(\frac{t}{2}-k\right)
\end{aligned}
$$

Here, $\psi^{i}$ is called a mother wavelet and the discrete filters $h(k)$ and $g(k)$ are quadrature mirror filters associated with the scaling function and the mother wavelet function [18].

The wavelet packet coefficients $c_{j, k}^{i}$ corresponding to the signal $f(t)$ can be obtained by Equation (12): $c_{j, k}^{i}=\int_{-\infty}^{+\infty} f(t) \psi_{j, k}^{i}(t) \mathrm{d} t$

The wavelet packet component of the signal at a particular node can be obtained by Equation (13):

$f_{j}^{i}(t)=\sum_{k=-\infty}^{+\infty} c_{j, k}^{i} \psi_{j, k}^{i}(t) \Delta t$

After performing the wavelet packet decomposition up to $j$ th level, the original signal can be represented as a summation of all wavelet packet components at jth level as shown in Equation (14):

$f(t)=\sum_{i=1}^{2^{j}} f_{j}^{i}(t)$

Based on the relation of frequency structure of wavelet decomposition, the frequency bandwidth of the approximation and detail of level $j$ are defined in Equations (15) and (16):

The frequency bandwidth of approximation:

$\left[0, \frac{1}{2} f_{s} 2^{-j}\right]$

The frequency bandwidth of detail:

$\left[\frac{1}{2} f_{s} 2^{-j}, \frac{1}{2} f_{s} 2^{-(j-1)}\right]$

where $f_{s}$ is the sampling rate.

\section{Wavelet-based methodology for acoustic emission signals analysis}

The base theory of wavelet was introduced in the previous section. It was understood that any signal can be decomposed into a set of wavelet component, each having its specific frequency range. In this work, for analyzing the AE signals, we focus on the energy criteria because of the different distribution of energy in each component that can be related to the specific failure mode. So, this idea can be the theoretical basis of the work discussed below.

If $f(t)$ is an AE signal based on Equation (12), it is possible to decompose the signal to wavelet components where $f_{j}^{1} \ldots f_{j}^{i}$ are the components of $j$ th level of the decomposed signal. According to the level of decomposed signal, $E_{j}^{1} \ldots E_{j}^{i}$ can be defined as the component energy at level $j$. This is mathematically expressed by Equation (17): 


$$
E_{j}^{i}(t)=\sum_{\tau=t_{0}}^{t}\left(f_{j}^{i}(\tau)\right)^{2}
$$

And the total energy of signal is defined by Equation (18):

$$
E_{\text {Total }}(t)=\sum_{j} E_{j}^{i}(t)
$$

In this work, the ratio of energies at different levels to the total energy is considered in order to find energy distribution displayed at different components. Let it be defined by Equation (19):

$$
P_{j}^{i}(t)=\frac{E_{j}^{i}(t)}{E_{\text {Total }}(t)}, \quad i=1 \ldots 2^{j}
$$

In this manner, $P_{j}^{i}(t)$ gives a relative energy distribution at each level.

\section{Results and discussion}

During a typical quasi-static loading of the composite material under mode I delamination, a large number of $\mathrm{AE}$ waveforms (Events) are recorded. Figure 4 shows the typical raw waveform of $\mathrm{AE}$ events in the time domain during the loading of composite material. These events have important roles in the analysis. In Figure 5, a load-time plot and its relationship with AE event energy for specimen $\mathrm{T} 3$ is shown. Completely different AE behavior was noticed where $\mathrm{AE}$ energies were first detected at the early stage of loading with alternating appearances during delamination. There is no event at the fist stage of loading and all the energy of loading is absorbed by the bending of the DCB specimen beams. But near the maximum load some

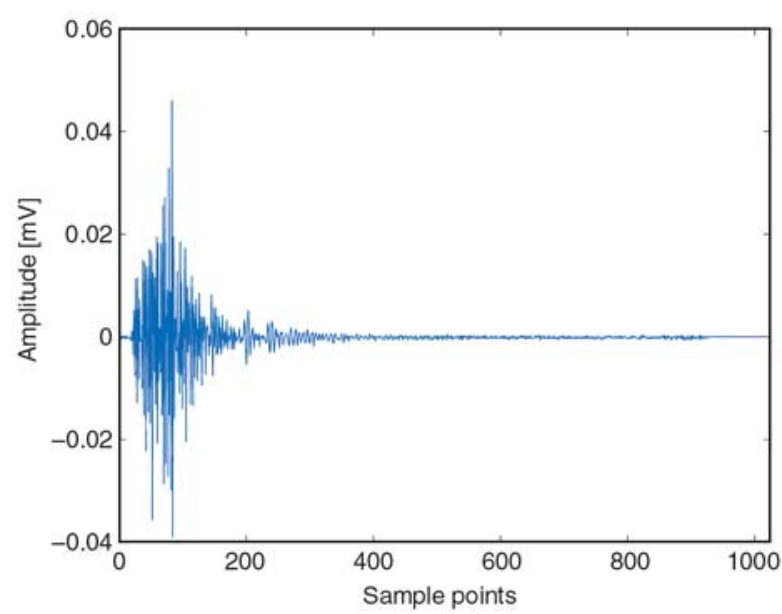

Figure 4. Typical AE waveform

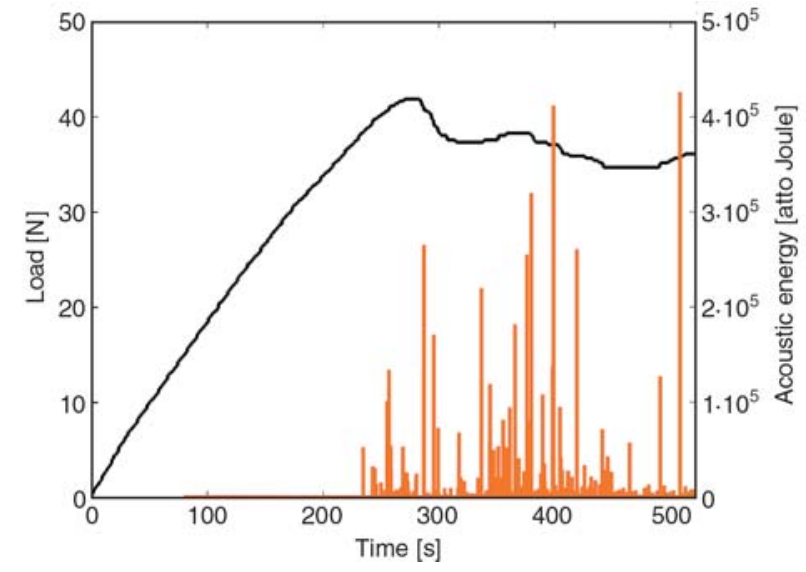

Figure 5. Delamination load and AE signal energy vs. time

$\mathrm{AE}$ events are detected and crack is being propagated.

For the analysis of waveforms, MATLAB software has been chosen since it has wavelet toolbox with numerous options and it is possible to create codes according to the application field. The code developed by the author was utilized to perform the wavelet based AE analysis. At first, we used the discrete wavelet transform as a rule of thumb to find an approximate range of energy concentration of signals. But, for the detailed information, we applied wavelet packet transform. In this way, wavelet detail components are also further decomposed to obtain its own approximation and detail. Because of splitting the wavelet detail component to its own approximation and detail, the frequency range is also split to two equal frequency ranges. By this technique, we can improve the relationship between the failure modes and corresponding frequency range. The existence of narrow band range of frequency in this method is the importance of this method rather than other wavelet transform methods which obtained the frequency range in wider range for the typical failure mode.

The wavelet packet transform (WPT) is applied to every signal. The application of wavelet packet analysis to the acquired $\mathrm{AE}$ signal results in its decomposition into three different levels of processed data. The mathematical criteria called entropy criteria $[16,22]$ are used to determine if certain decomposition is sufficient or more levels are needed. There are a lot of wavelet families that were used in the analysis, one of which was called Daubechies' wavelet $[18,19]$, and was applied in the current work. The detail results are obtained in 

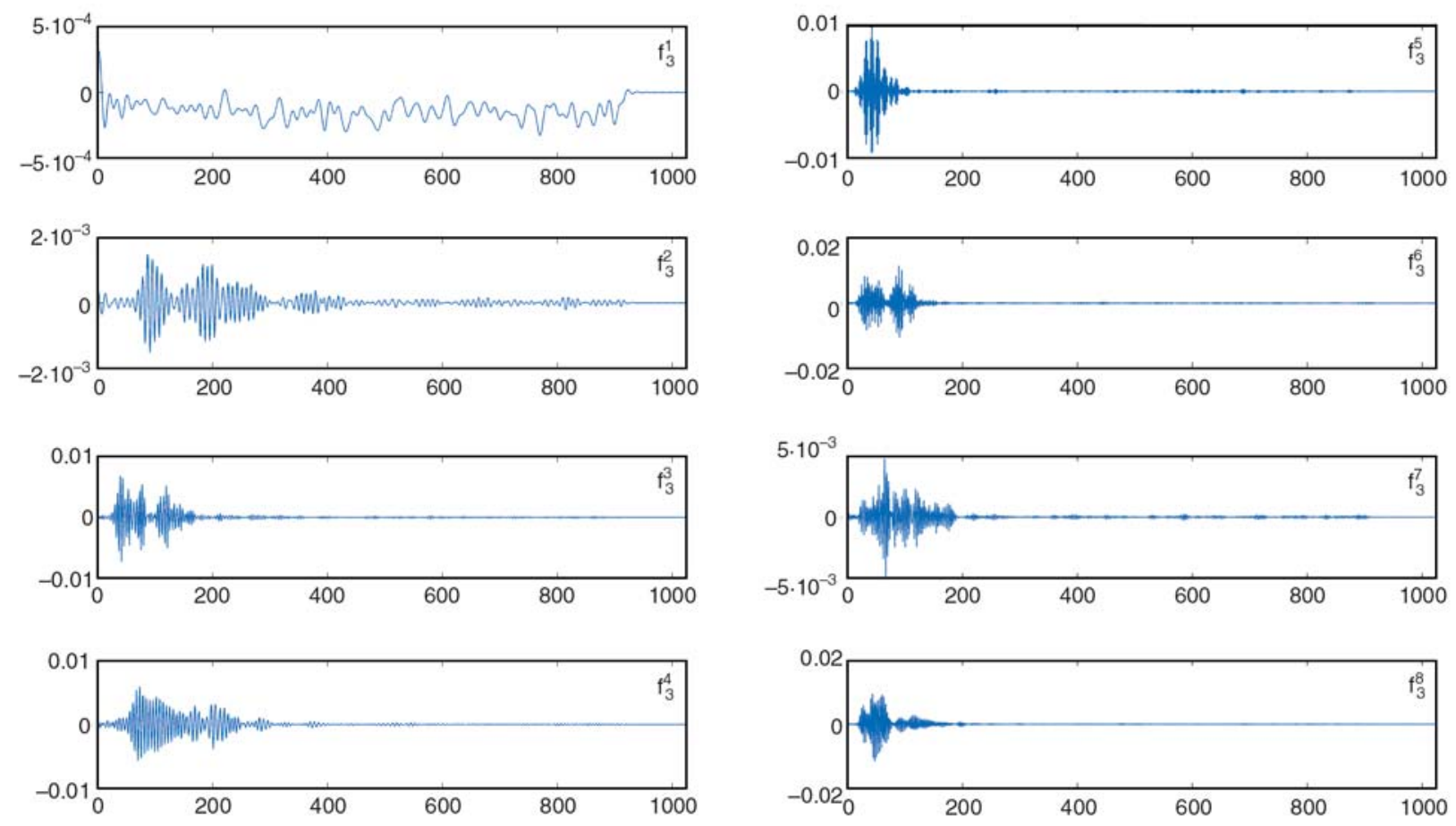

Figure 6. Three level wavelet packet decomposition of an AE signal for T3 (amplitude in [mV] vs. sample points)

the next plots. The full decomposition of each signal by the wavelet packet transform is carried out and all the components of the third level were only used to find the dominant energy levels. Figure 6 shows the three level wavelet packet decomposition of AE signals for specimen T3. Each component at level 3 represents a specific frequency range, and the frequency range increases with
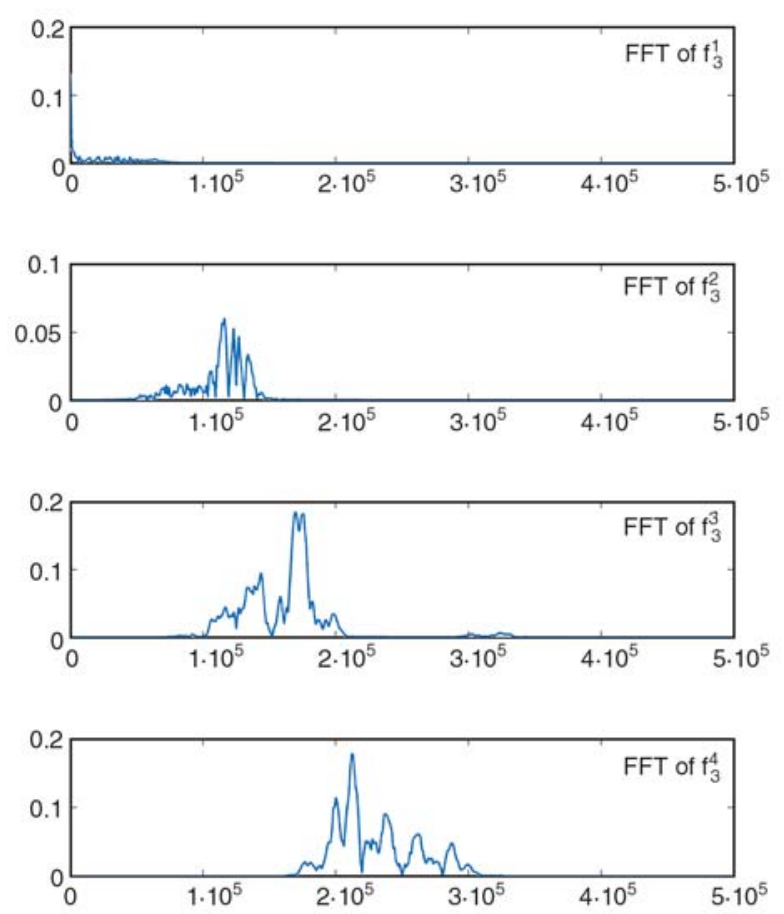

increasing the wavelet levels. There are eight components at third level whose frequency range is obtained from Equations (15) and (16). The FFT method is used to obtain the frequency content of the decomposed signals. Figure 7 is the frequency content of each component of decomposed signals that have been shown in Figure 6. From the graphs it is obvious that the frequency range of the decom-
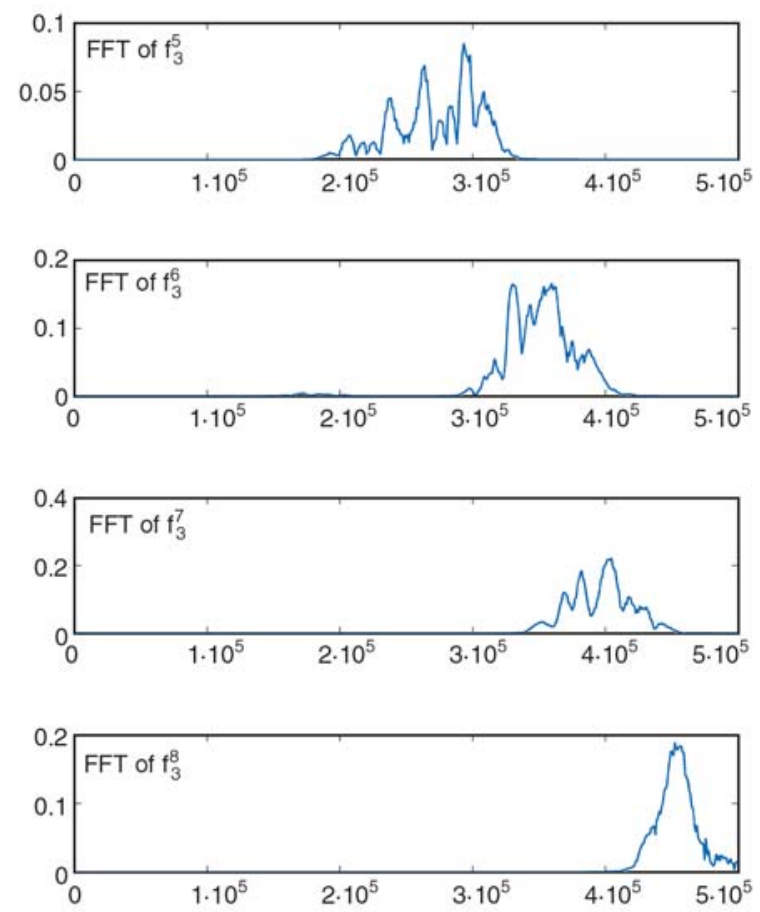

Figure 7. FFT of the decomposed components of level 3 (FFT amplitude [mV2/Hz] vs. frequency [Hz]) 

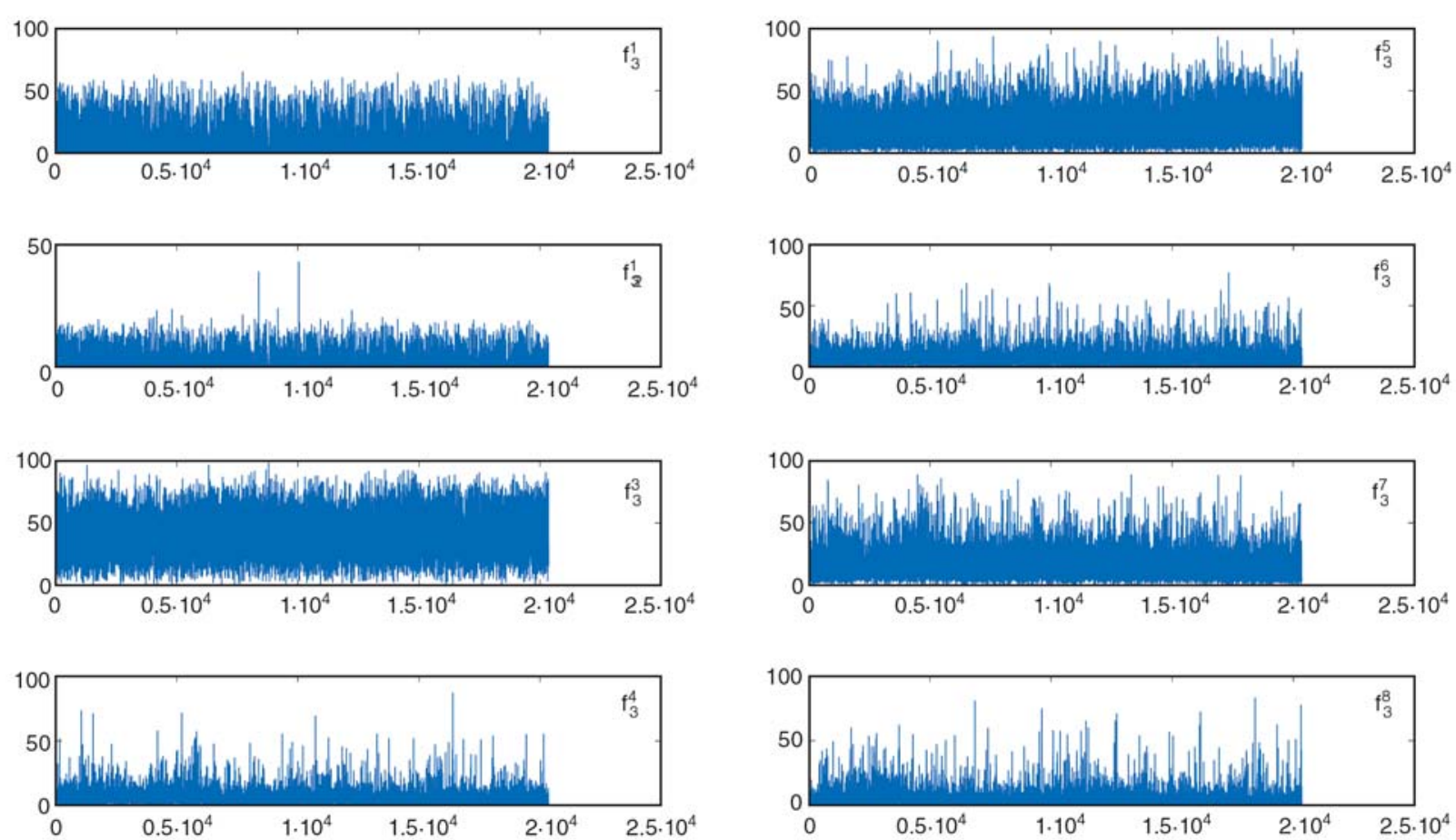

Figure 8. Energy percentage of each component of level 3 for T3 (energy [\%] vs. waveform number)

posed components, increased from one component to another.

According to the Nyquist sampling theorem, the signal must be sampled at twice the highest frequency contained in the signal. Therefore, in this case, the sampling frequency set to $1 \mathrm{MHz}$ and the transformed signals frequency must be up to
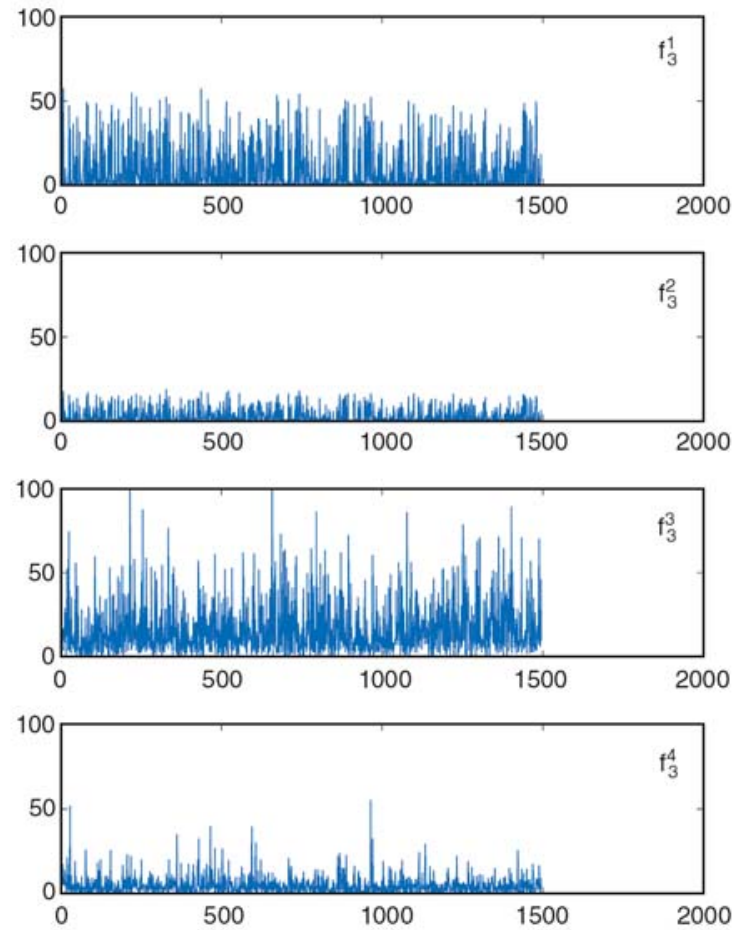

$500 \mathrm{kHz}$. The frequency interval between the two adjacent components is $62.5 \mathrm{kHz}$, according to Equations (15) and (16).

From the methodology described in the previous section, after the decomposition of all AE signals, the energy percentage of the signal in each component is compared with the total energy of the $\mathrm{AE}$
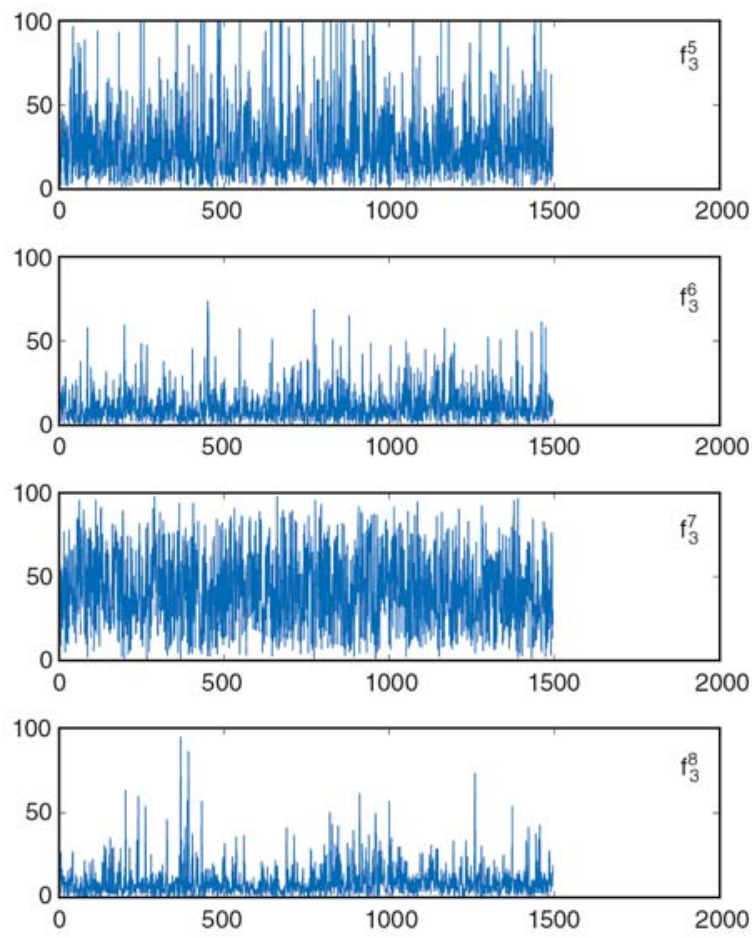

Figure 9. Energy percentage of each component of level 3 for T5 (energy [\%] vs. waveform number) 
signal that can be calculated based on Equations (17), (18) and (19). In Figures 8 and 9, the results of the calculation to find the energy distribution in each component, are obtained for specimens $\mathrm{T} 3$ and T5, respectively. In these figures, the vertical coordinate is the energy and the horizontal coordinate is the waveform number.

The variation of distribution is different from one component to another one. The greatest percentage of the energy is distributed in 3 components that called $f_{3}^{3}, f_{3}^{5}, f_{3}^{7}$. For interface $\mathrm{T} 3$ the highest energy is concentrated at component $f_{3}^{3}$ while for interface T5 the highest energy is related to component $f_{3}^{7}$. Obviously, at the same component for different interfaces, the energy distribution pattern is different. This difference could be directly related to different damage mechanisms during mode I delamination. It was concluded that there are two separate dominant damage mechanisms in interfaces T3 and T5. From Figure 7 the frequency range of three dominant components is obtainable: for component $f_{3}^{3}$ the frequency range is at $125-200 \mathrm{kHz}$, component $f_{3}^{5}$ at $250-310 \mathrm{kHz}$ and component $f_{3}^{7}$ at $375-$ $440 \mathrm{kHz}$, approximately. For interface T3 components $f_{3}^{3}, f_{3}^{5}$ are dominant between the three components while for interface T5 $f_{3}^{7}$ is dominant. Also, based on the energy distribution at each component, in this work we additionally calculated the average energy of each component. The results show that for interface $\mathrm{T} 3, f_{3}^{3}$ possesses about $40 \%$ of total energy, $f_{3}^{5}$ about $20 \%$ and $f_{3}^{7} 15 \%$ in average. These values for interface T5 are $f_{3}^{3}=15 \%$, $f_{3}^{5}=25 \%$ and $f_{3}^{7}=40 \%$. Summary of wavelet packet results for dominant components are shown in Table 1.

To understand the relationship between dominant component frequency and failure mode, microscopic observation is considered, too. Damage mechanisms such as fiber/matrix debonding and

Table 1. Summary of wavelet packet analysis for dominant components

\begin{tabular}{|c|c|c|}
\hline Specimen & $\begin{array}{c}\text { Energy percentage of } \\
\text { dominant components } \\
{[\%]}\end{array}$ & $\begin{array}{c}\text { Frequency range of } \\
\text { dominant components } \\
{[\mathbf{k H z}]}\end{array}$ \\
\hline \multirow{3}{*}{ T3 } & $f_{3}^{3}=40$ & $125-250$ \\
\cline { 2 - 3 } & $f_{3}^{5}=20$ & $250-310$ \\
\cline { 2 - 3 } & $f_{3}^{7}=15$ & $375-440$ \\
\hline \multirow{3}{*}{ T5 } & $f_{3}^{3}=15$ & $125-250$ \\
\cline { 2 - 3 } & $f_{3}^{5}=25$ & $250-310$ \\
\cline { 2 - 3 } & $f_{3}^{7}=40$ & $375-440$ \\
\hline
\end{tabular}
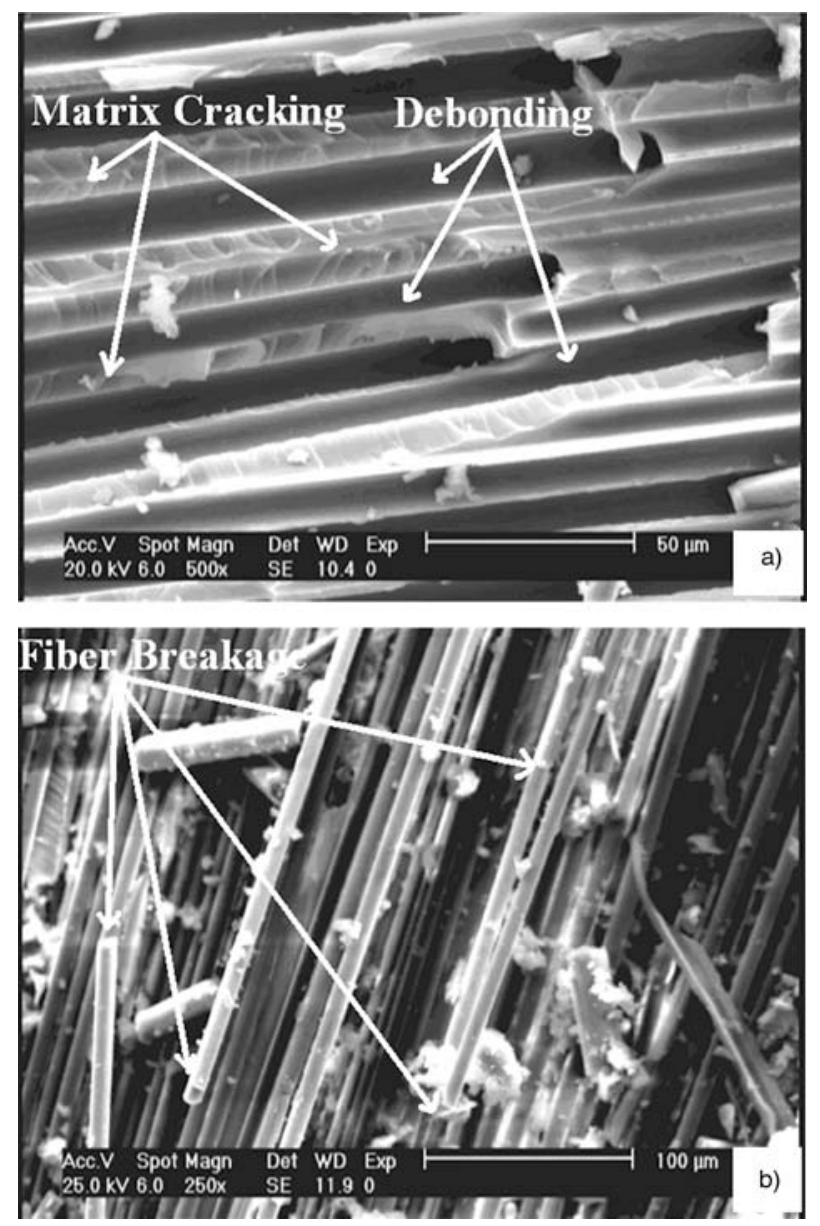

Figure 10. SEM observation of dominant failure mechanisms during mode I delamination: a) matrix cracking and debonding for $\mathrm{T} 3$ specimen, $\mathrm{b}$ ) fiber breakage for $\mathrm{T} 5$ specimen

matrix cracking have been observed by SEM microscope (Figure 10a). The break of fiber bridges is observed, especially in unidirectional composites with the weak fiber-matrix adhesion [23, 24]. Its AE energy contribution is negligible in mode I delamination propagation where the woven/woven mid plane is used. On the other hand for interface $\mathrm{T} 3$ only matrix cracking and debonding between fiber/ matrix are considered and a little fiber breakages have been observed. As observed from SEM results, matrix cracking and debonding are significant failure mechanisms during mode I delamination in the woven composites, while for interface T5 fiber breakage and debonding were significant too (Figure 10b). But what is the relation between three dominant components frequency range and SEM observation?

The possibility to distinguish between the matrix cracking and interface debonding relies on different visco-elastic relaxation processes near to the source 
itself [25]. Intrinsic frequencies $f_{i}$ and elastic acoustic velocities $c_{i}$ of the relaxation processes differ in the fiber, matrix and matrix/fiber interface because of the correlation with the relaxation times $\tau_{i}$, elastic module $E_{i}$ and densities $\rho_{i}$ according to Equation (20) [25]:

$f_{i} \sim \frac{1}{\tau_{i}} \sim c_{i} \sim \sqrt{\frac{E_{i}}{\rho_{i}}}$

Thus, the polyester-matrix cracking creates lower wide-band frequencies than the glass-fiber/matrix interface debonding.

From the above discussion it is concluded that component $f_{3}^{3}$ at low frequency $(125-200 \mathrm{kHz})$ is associated with matrix cracking. Another dominant wavelet component at level $3, f_{3}^{5}$ can be directly corresponded to the other damage mechanisms e.g. debonding between matrix and fiber where the frequency range is $250-310 \mathrm{kHz}$ [13]. The above argument is consistent with the existing research results based on the AE frequency contents of signals [11-13]. Finally, the last one, that exists other source in the signal with high frequency (375$440 \mathrm{kHz}$ ) dominated by component, $f_{3}^{7}$, representing fiber breakage failure mode [13, 25]. Also the SEM observation is in agreement with these results as obtained from wavelet analysis.

In interface T5 fiber breakage was more significant and $40 \%$ of energy was distributed in fiber breakage range due to the existence of Fiber Bridge in unidirectional interface. But in woven interface, fiber length is not long enough to be bridged, so instead of fiber breakage, fiber debonding is dominant. However, debonding is detected for both of interfaces but the portion of energy for fiber breakage was higher for interface $\mathrm{T} 5$.

Consequently, the other components in wavelet analysis can be considered as a noise, fiber pull-out and sliding of fiber.

\section{Conclusions}

The objective of this study is to investigate the mode I delamination by AE technique and determine the relationship between $\mathrm{AE}$ results and damage mechanisms in mode I delamination. A wavelet packet transform methodology was established for the post-processing of the $\mathrm{AE}$ waveforms recorded during the testing of a composite material. Also,
SEM observation of DCB specimens shows that the failure modes, such as matrix cracking, debonding and fiber breakage, were the sources of AE signals but which frequency range is in relation with these failure modes that is the objective that clarified by wavelet based method.

The component energy of a decomposed AE signal provides a comprehensive means of identifying $\mathrm{AE}$ sources. The energy concept shows different potential failure modes of a specimen by discriminating the energy quantity and frequency range.

As a result, from the energy concentration criterion, it was found that three dominant components at the frequency ranges of 125-200, 250-310 and 375$440 \mathrm{kHz}$ are concerned with the matrix cracking, debonding and fiber breakage, respectively.

The energy pattern was different from one specimen to another one due to different damage mechanisms. This is the ability of wavelet analysis that can be used as an effective tool for monitoring and characterizing the failure modes especially in the composite materials.

\section{Acknowledgements}

A part of this research is financially supported by the Pars Gas and Oil Company of Iran. The authors wish to thank the Department of Mechanical Engineering at Amirkabir University of Technology for providing the facilities to carrying out the tests.

\section{References}

[1] ASTM D5528: Standard test method for mode I interlaminar fracture toughness of unidirectional fiberreinforced polymer matrix composites (1994).

[2] Pappas Y. Z., Markopoulos Y. P., Kostopoulos V.: Failure mechanisms analysis of 2D carbon/carbon using acoustic emission monitoring. NDT\&E International, 31, 157-163 (1998). DOI: $\underline{10.1016 / S 0963-8695(98) 00002-4}$

[3] Velmurugan R., Solaimurugan S.: Improvements in mode I interlaminar fracture toughness and in-plane mechanical properties of stitched glass/polyester composites. Composites Science and Technology, 67, 6169 (2007).

DOI: 10.1016/j.compscitech.2006.03.032

[4] Benevolenski O. I., Karger-Kocsis J., Czigány T., Romhány G.: Mode I fracture resistance of glass fiber mat-reinforced polypropylene composites at various degree of consolidation. Composites Part A: Applied Science and Manufacturing, 34, 267-273 (2003). DOI: $\underline{10.1016 / \mathrm{S} 1359-835 \mathrm{X}(02) 00045-3}$ 
[5] Romhány G., Szebényi G.: Interlaminar crack propagation in MWCNT/fiber reinforced hybrid composites. Express Polymer Letters, 3, 145-151 (2009). DOI: $10.3144 /$ expresspolymlett.2009.19

[6] Benmedakhene S., Kenane M., Benzeggagh M. L.: Initiation and growth of delamination in glass/epoxy composites subjected to static and dynamic loading by acoustic emission monitoring. Composites Science and Technology, 59, 201-208 (1999).

DOI: $10.1016 / \mathrm{S} 0266-3538(98) 00063-3$

[7] Godin N., Huguet S., Gaertner R.: Integration of the Kohonen's self-organising map and k-means algorithm for the segmentation of the AE data collected during tensile tests on cross-ply composites. NDT\&E International, 38, 299-309 (2005).

DOI: $\underline{10.1016 / \text { j.ndteint.2004.09.006 }}$

[8] Philippidis T. P., Nikolaidis V. N., Anastassopoulos A. A.: Damage characterization of carbon/carbon laminates using neural network techniques on AE signals. NDT\&E International, 31, 329-340 (1998). DOI: $10.1016 / \mathrm{S} 0963-8695(98) 00015-2$

[9] de Oliveira R., Marques A. T.: Health monitoring of FRP using acoustic emission and artificial neural networks. Computers and Structures, 86, 367-373 (2008).

DOI: $10.1016 /$ j.compstruc.2007.02.015

[10] Siron O., Chollon G., Tsuda H., Yamauchi H., Maeda K., Kosaka K.: Microstructural and mechanical properties of filler-added coal-tar pitch-based C/C composites: The damage and fracture process in correlation with AE waveform parameters. Carbon, 39, 20652075 (2001). DOI: $10.1016 / \mathrm{S} 0008-6223(99) 00270-5$

[11] Ramirez-Jimenez C. R., Papadakis N., Reynolds N., Gan T. H., Purnell P., Pharaoh M.: Identification of failure modes in glass/polypropylene composites by means of the primary frequency content of the acoustic emission event. Composites Science and Technology, 64, 1819-1827 (2004).

DOI: 10.1016/j.compscitech.2004.01.008

[12] Haselbach W., Lauke B.: Acoustic emission of debonding between fibre and matrix to evaluate local adhesion. Composites Science and Technology, 63, 2155-2162 (2003). DOI: $10.1016 / \mathrm{S} 0266-3538(03) 00193-3$

[13] Ni Q-Q., Iwamoto M.: Wavelet transform of acoustic emission signals in failure of model composites. Engineering Fracture Mechanic, 69, 717-728 (2002). DOI: 10.1016/S0013-7944(01)00105-9
[14] Qi G., Barhorst A., Hashemi J., Kamala G.: Discrete wavelet decomposition of acoustic emission signals from carbon-fiber-reinforced composites. Composites Science and Technology, 57, 389-403 (1997). DOI: $10.1016 / \mathrm{S} 0266-3538(96) 00157-1$

[15] Qi G.: Wavelet-based AE characterization of composite materials. NDT\&E International, 33, 133-144 (2000).

DOI: $10.1016 / \mathrm{S} 0963-8695(99) 00037-7$

[16] Loutas T. H., Kostopoulos V., Ramirez-Jimenez C., Pharaoh M.: Damage evolution in center-holed glass/polyester composites under quasi-static loading using time/frequency analysis of acoustic emission monitored waveforms. Composites Science and Technology, 66, 1366-1375 (2006).

DOI: 10.1016/j.compscitech.2005.09.011

[17] Velayudham A., Krishnamurthy R., Soundarapandian T.: Acoustic emission based drill condition monitoring during drilling of glass/phenolic polymeric composite using wavelet packet transform. Materials Science and Engineering: Part A, 412, 141-145 (2005). DOI: $10.1016 /$ j.msea.2005.08.036

[18] Rao R. M., Bopardikar A. S.: Wavelet transforms introduction to theory and applications. Addison-Wesley, Boston (1998).

[19] Soman K. P., Ramachandran K. I.: Insight into wavelets from theory to practice. Prentice-Hall, New Delhi (2004).

[20] Wojtaszczyk P.: A mathematical introduction to wavelets. Cambridge University Press, Cambridge (1997).

[21] Walnut D. F.: An introduction to wavelet analysis. Birkhäuser, Boston (2002).

[22] Coifman R. R., Wickerhauser M. V.: Entropy-based algorithms for best basis selection. IEEE Transactions on Information Theory, 38, 713-718 (1992). DOI: $\underline{10.1109 / 18.119732}$

[23] Brunner A. J., Blackman B. R. K., Williams J. G.: Calculating a damage parameter and bridging stress from GIC delamination tests on fibre composites. Composites Science and Technology, 66, 785-795 (2006). DOI: $10.1016 /$ j.compscitech.2004.12.040

[24] de Moraisa A. B., Marques A. T.: Mode I interlaminar fracture of carbon/epoxy multidirectional laminates. Composites Science and Technology, 64, 2261-2270 (2004). DOI: 10.1016/j.compscitech.2004.03.001

[25] Bohse J. Acoustic emission characteristics of microfailure processes in polymer blends and composites. Composites Science and Technology, 60, 1213-1226 (2000). DOI: $10.1016 / \mathrm{S} 0266-3538(00) 00060-9$ 